Józef Stala

The Pontifical University of John Paul II in Krakow, Poland

\title{
Konferenzbericht
}

\section{Współczesna rodzina: perspektywy lokalne i europejskie $X$ międzynarodowa konferencja naukowa: \\ (Die Familie heute: lokale und europäische Perspektiven Zehnte internationale Wissenschaftskonferenz), Krakau, 23. bis 24. Oktober 2013}

Am 23. und 24. Oktober fand im Gebäude der Zentralbibliothek der Päpstlichen Universität Johannes Paul II. in Krakau (UPJPII) die zehnte internationale Wissenschaftskonferenz Wspótczesna rodzina: perspektywy lokalne i europejskie (Die Familie heute: lokale und europäische Perspektiven) unter der Schirmherrschaft von Seiner Magnifizenz H. H. Prof. Dr. habil. Władysław Zuziak, Rektor der UPJPII, statt. Die Organisation der Konferenz lag in den Händen von H. H. Prof. Dr. habil. Józef Stala (Theologische Fakultät, Sektion Tarnów, der UPJPII) und Dr. habil. Elżbieta Osewska (Fakultät für Familienwissenschaften der Universität Kardinal Stefan Wyszyński in Warschau [UKSW]), die mit H. H. Prof. Dr. habil. Janusz Mastalki und Dr. habil. Małgorzata Duda (Fakultät für Sozialwissenschaften der UPJPII) sowie mit H. H. Dr. Mariusz Kuźniar und Dr. Noemi Bravena (Katholisch-Theologische Fakultät der Karls-Universität in Prag, Tschechien), Prof. Dr. Janez Vodičar, H. H. Prof. Dr. Stanko Gerjolj (Theologische Fakultät der Universität in Ljubljana, Slowenien) zusammen arbeiteten. Die Organisation der Konferenz erfolgte im Rahmen der Krakowskich Konferencji Naukowych (Krakauer Wissenschaftskonferenzen), finanziert wurde sie durch die Stadt Krakau wie auch durch die Stiftung im Namen der hl. Königin Jadwiga für die UPJPII in Krakau, durch die Theologische Fakultät, Sektion Tarnów, der UPJPII und durch weitere Spenden von Förderern der Wissenschaft. Über die Konferenz berichteten die Krakauer Zeitung, die 
Redaktion für Zeitchronik von TVP Krakau, Dziennik Polski (Polnische Tageszeitung) sowie die Rundfunkstationen Radio PLUS und Radio Bonus.

An der Konferenz nahmen Vertreter aller kirchlichen Fakultäten in Polen sowie drei von sechs, ,weltlichen" Fakultäten teil, die Forschungsarbeiten zur Familienwissenschaft durchführen, daneben auch Studenten, Lehrer, Erzieher, Eltern und weitere Personen, die sich für die aufgegriffene Problematik interessieren. Insgesamt konnten etwa 200 Konferenzteilnehmer gezählt werden: Am ersten Tag beteiligten sich über 160 Interessierte (aus Polen, Slowenien, Deutschland, Tschechien und der Slowakei) an den Beratungen, am zweiten (nicht öffentlichen) Tag über 30 Vertreter von Universitäten in Polen und im Ausland (aus Tschechien, Slowenien und der Slowakei), die Forschungen im Bereich der Familienwissenschaften betreiben.

Darüber hinaus ging eine Vielzahl von Glückwunschbriefen ein: H. H. Prof. Dr. habil. Władysław Zuziak (Rektor UPJPII), Bischof Dr. Jan Wątroba (Bischof von Rzeszów, Vorsitzender der Kommission für Ehe und Familie der Polnischen Bischofskonferenz), Bischof Dr. Andrzej Jeż(Bischof von Tarnów), Bischof Karel Herbst (Weihbischof in der Erzdiözese Prag, Vorsitzender der Kommission für Katechese der Tschechischen Bischofskonferenz), Elizabeth Davies (Bevollmächtigte für Ehe- und Familienprogramme der Englischen und Walisischen Bischofskonferenz), H. H. Prof. Dr. habil. Tadeusz Dola (Dekan der Theologischen Fakultät der Universität in Oppeln), H. H. Prof. UWM Dr. habil. Piotr Duksa (Dekan der Theologischen Fakultät der Universität Ermland-Masuren), H. H. Prof. UMK Dr. habil. Dariusz Kotecki (Dekan der Theologischen Fakultät der Nikolaus-Kopernikus-Universität in Toruń), H. H. Dr. habil. Antoni Bartoszek (Dekan der Theologischen Fakultät der Schlesischen Universität in Kattowitz), H. H. Prof. UPJPII Dr. habil. Wojciech Zyzak (Dekan der Theologischen Fakultät der UPJPII), H. H. Prof. UPJPII Dr. habil. Ireneusz Stolarczyk (Dekan der Theologischen Fakultät, Sektion Tarnów, der UPJPII), H. H. Prof. Dr. habil. Christian Gostečnik (Dekan der Theologischen Fakultät der Universität Ljubljana), H. H. Dr. Prokop Broż (Dekan der Katholisch-Theologischen Fakultät der Karls-Universität in Prag), H. H. Prof. Dr. Hektor Scerri (Prodekan der Theologischen Fakultät der Universität Malta), H. H. Prof. UKSW Dr. habil. Mieczysław Ozorowski (Dekan der Fakultät für Familienwissenschaften der UKSW und Vorsitzender der Polnischen Vereinigung für Familienforschung), H. H. Prof. Dr. habil. Piotr Morciniec (Vorsitzender der Sektion Polen und Präsidiumsmitglied der Europäischen Gesellschaft für Katholische Theologie). 
$\mathrm{Zu}$ Beginn der Konferenz begrüßte H. H. Prof. Dr. habil. Józef Stala die Gäste in polnischer und deutscher Sprache, bevor er in die Thematik der Beratungen einführte. Er verwies außerdem auf das Engagement der einzelnen Personen, die sich wesentlich bei der Vorbereitung der Konferenz eingebracht haben und dankte den Mitarbeitern der eigenen Universität ausdrücklich für ihre tatkräftige Unterstützung: Iwona Bąk vom Büro für wissenschaftliche Forschungen an der UPJPII; Magdalena Nagięć, Direktorin der Bibliothek der UPJPII, zusammen mit ihrem gesamten Team; Sebastian Wojnowski, Direktor des Wissenschaftsverlags der UPJPII, und seinem Mitarbeiterstab; dem Büro für Promotion und Information an der UPJPII mit seinem Leiter Krzysztof Mleczko und der Pressebeauftragten Monika Wiertek; dem Administrator der Internetseite Piotr Kowalski. Anschließend unterstrichen H. H. Prof. Dr. habil. Władysław Zuziak - Rektor der UPJPII, H. H. Dr. Antoni Świerczek, Prodekan der Fakultät für Sozialwissenschaften an der UPJPII und Prof. Dr. habil. Kazimierz Wiatr, Senator der Volksrepublik Polen, in ihren Grußworten die Bedeutung, Aktualität und Dringlichkeit dieser Konferenz.

Der erste Konferenztag untergliederte sich in zwei Teile: Im ersten, den H. H. Prof. Dr. habil. Józef Stala leitete, waren die folgenden vier Hauptvorträge zu hören: 1. H. H. Prof. Dr. Stanko Gerjolj (Universität Ljubljana, Slowenien), Biblical Families and Current Educational Challenges (Biblische Familien und aktuelle Herausforderungen für die Erziehung); 2. H. H. Dr. habil. Zbigniew Waleszczuk (Katholische Universität Eichstätt-Ingolstadt, Deutschland), Die Familie - ein Relikt aus der Vergangenheit oder das Fundament der Zukunft?; 3. H. H. Prof. Dr. habil. Anton Fabian (Pavol-Jozef-Šafárik-Universität Košice), Rozdiely v interpretacii manzelstva v súcasnej spolocnosti a cirkvi (Aktuelle Unterschiede bei der Interpretation der Ehe durch Gesellschaft und Kirche); 4. H. H. Prof. Dr. habil. Wojciech Misztal (UPJPII), Matriarchat, patriarchat czy taka sama godność $i$ różnorodnosśc zadań? Przyczynek do oceny aktualności chrześcijańskiej duchowości rodziny (Matriarchat, Patriarchat - Gleichwertigkeit trotz verschiedener Aufgaben? Ein Beitrag zur Einschätzung, wie aktuell die christliche Geistlichkeit der Familie ist). Die Vertreter von vier europäischen Universitäten, die auf biblische Quellen, auf Lehrschreiben der Kirche und auf aktuelle staatliche Dokumente Bezug nahmen, verwiesen auf die Familie als grundlegendes Umfeld für die Entwicklung und die Erziehung des Menschen. Sie betonten, dass eine Minimierung des Wertes von Familie in der Konsequenz die Schwächung des Menschen selbst, der Gesellschaft, der 
Nation und der Kirche nach sich zieht. Unmittelbar auf die Vorträge folgte die Diskussion der aufgestellten Thesen.

Im weiteren Verlauf des Tages hatten die Teilnehmer die Möglichkeit, sich an einer von drei parallel stattfindenden Themengruppen zu beteiligen. In der ersten Gruppe Kościót a rodzina (Kirche und Familie), die H. H. Prof. Dr. habil. Józef Stala leitete, wurden die folgenden sieben Referate vorgetragen: 1. H: H. Dr. habil. Marek Kluz (UPJPII), Problem wychowania moralnego wyzwaniem dla wspótczesnej rodziny (Das Problem der Moralerziehung als Herausforderung für die Familie von heute); 2. Dr. Katarzyna Zielińska-Król (KUL), H. H. Dr. Jerzy Król (PWSZ Chełm), Perspektywy wychowawcze współczesnej rodziny (Perspektiven für die Erziehung in der Familie der Gegenwart); 3. Dr. Łukasz Ryszka (UPJPII), Rola Kościoła katolickiego w umacnianiu świadomości narodowo-kulturowej Polaków w Niemczech (Die Rolle der katholischen Kirche bei der Festigung des national-kulturellen Bewusstseins der Polen in Deutschland); 4. Magister lic. Karina Zając (UŚ), Rodzina śląka: co zostało z tradycji? Perspektywy odnowy (Die schlesische Familie: Was wurde aus der Tradition? Perspektiven der Erneuerung); 5. H. H. Dr. Cezary Naumowicz (UKSW), Rola rodziny $w$ budowaniu cywilizacji pokoju - kontekst lokalny i globalny (Die Rolle der Familie beim Aufbau einer Zivilisation des Friedens - lokaler und globaler Kontext); 6. H. H. Robert Kantor (UPJPII), Malżeństwo kanoniczne jako budowanie wspólnoty rodzinnej na dobre i na złe do końca życia (Perspektywa sadowa wykluczenia nierozerwalności matżeństwa) (Die nach kanonischem Recht geschlossene Ehe, auf der die familiäre Gemeinschaft in guten und schlechten Tagen bis zum Ende des Lebens aufbaut [Die rechtliche Perspektive der Unauflösbarkeit der Ehe]); H. H. Prof. Dr. habil. Józef Stala (UPJPII), Troska Kościoła o wspótczesna rodzinę, (Die Sorge der Kirche um die moderne Familie). An das letzte Referat schlossen sich eine Diskussion und ein lebhafter Erfahrungsaustausch rund um den erörterten Themenbereich an.

Dagegen waren in der zweiten, von H. H. Dr. Antoni Świerczek geleiteten Themengruppe: Rodzina wobec problemów (Die Familie angesichts ihrer Probleme) folgende sieben Referate zu hören: 1. H. H. Dr. Mariusz Kuźniar (Karls-Universität in Prag), Hodnoty a životni styl českých rodin - sociologický výzkum (Werte und der Lebensstil christlicher Familien - soziologische Untersuchungen); 2. H. H. Prof. UPJPII Dr. habil. Michał Drożdż (UPJPII), Rodzina wobec medialnej „,produkcji sensu” (Die Familie angesichts der medialen „Sinnproduktion”); 3. H. H. Dr. habil. Dariusz 
Lipiec (KUL), Rodzina człowieka niepetnosprawnego jako wyzwanie dla duszpasterstwa parafialnego (Die Familie mit einem behinderten Menschen als Herausforderung für die Seelsorge in der Pfarrgemeinde); 4. Dr. Jolanta Baran (UP Krakau), O wybranych obszarach wsparcia rodzin wychowujacych dziecko $z$ uszkodzonym stuchem - fakty i prognozy (Ausgewählte Unterstützungsmöglichkeiten für Familien mit einem schwerhörigen Kind - Fakten und Prognosen); 5. H. H. Dr. Grzegorz Godawa (UPJPII), Psychospołeczne uwarunkowania wychowania dziecka przez samotnego ojca. Sytuacja w Polsce i Wielkiej Brytanii (Psychosoziale Bedingungen der Erziehung von Kindern, die beim Vater leben. Die Situation in Polen und Großbritannien); 6. Mag. Ewa Kiełek-Rataj(UO), Rodzicielstwo wnarracjach osób wybierajacych bezdzietny styl życia (Elternschaft in Erzählungen von Menschen, die ein Leben ohne Kinder wählen); 7. Dr. Noemi Bravená (Karls-Universität Prag), Současná česká rodina a péče o presah dítěte (Die tschechische Familie der Gegenwart und ihre Sorge um die transzendente Dimension der Kindererziehung). Die Beratungen schlossen mit einer Vielzahl an Vorschlägen, die während der Diskussion und im Erfahrungsaustausch geäußert wurden.

In der dritten Themengruppe: Rodzina $w$ perspektywie międzynarodowej (Die Familie in der internationalen Perspektive) wurden unter der Leitung von Prof. UKSW Dr. habil. Elżbieta Osewska die folgenden acht Fragenkreise dargelegt: 1. H. H. Prof. Dr. habil. Janez Vodičar (Universität Ljubljana, Slowenien), Married Couples Ministries as a Place of Catechesis (Die Seelsorge für Ehepaare als Ort der Katechese); 2. Dr. Barbara Simonič (Dozentin an der Universität Ljubljana, Slowenien), Empathic Parenting and Child Development (Empathische Elternschaft und die Entwicklung des Kindes); 3. H. H. Dr. habil. Ludvík Dřímal (Dozent an der Karls-Universität in Prag), Katecheze v současné české rodině (Katechese in der tschechischen Familie der Gegenwart); 4. Mag. Tanja Pate (Universität Ljubljana, Slowenien), Families with Chronically Ill Children and Relational Family Model (Familien mit chronisch kranken Kindern und ein beziehungsgestütztes Familienmodell); 5. Dr. Maria Sedlakova (Košice, Slowakei), Zmysel zivota nahradnych rodicov (Die Frage nach dem Sinn des Lebens bei Pflegeltern); 6. Mag. Iwona Waldzińska (PWSZ Auschwitz), A Migration Profile of a Polish Family after Poland's Accession to the European Union (Das Migrationsprofil der polnischen Familie nach dem Beitritt Polens zur Europäischen Union); 7. Prof. UKSW Dr. habil. Elżbieta Osewska(UKSW), The Family: A Potential 
Space for Religious Experience (Die Familie: Ein potenzieller Ort für religiöse Erfahrung); 8. Prof. Ateneum Dr. habil. Małgorzata Stopikowska (Ateneum - Hochschule Danzig), Małżeństwa polsko-arabskie: specyfika i zagrożenia (Das polnisch-arabische Ehepaar: Spezifika und Gefahren). Ähnlich wie in den beiden anderen Gruppen endeten die Beratungen mit einem interessanten Gedankenaustausch und einer in englischer und polnischer Sprache geführten Diskussion.

Am zweiten Konferenztag, der sich ebenfalls in zwei Teile gliederte, nahmen Hochschullehrer - Vertreter der kirchlichen und weltlichen Fakultäten teil, die sich wissenschaftlich mit derFamilie befassen. Für den ersten, von H. H. Prof. Dr. habil. Piotr Morciniec geleiteten Teil Nauki o rodzinie w kontekście aktualnych wyzwań społeczno-politycznych, kulturowych, religijnych $i$ edukacyjnych (Familienwissenschaften im Kontext der aktuellen gesellschaftspolitischen, kulturellen, religiösen und erzieherischen Herausforderungen), waren folgende Referenten geladen:1. H. H. Prof. Dr. habil. Bronisław Mierzwiński (UKSW), Perspektywa teologiczna (Die theologische Perspektive); 2. H. H. Prof. Dr. habil. Ireneusz Mroczkowski (UKSW), Perspektywa antropologiczna (Die anthropologische Perspektive); 3. H. H. Prof. Dr. habil. Janusz Mastalski (UPJPII), Perspektywa pedagogiczna (Die pädagogische Perspektive); 4. Dr. habil. Alicja Kalus (UO), Perspektywa psychologiczna (Die psychologische Perspektive); 5. Prof. Dr. habil. Lucjan Kocik (UPJPII), Perspektywa socjologiczna (Die soziologische Perspektive). Auf die Darstellung der fünf Perspektiven hinsichtlich der Familienwissenschaften folgte eine Diskussion, in der auf die Notwendigkeit verwiesen wurde, dauerhafte Fundamente für die Familienwissenschaften aufzuzeigen.

Für den anschließenden zweiten Teil Nauki o rodzinie - miejsce nauk o rodzinie wśród innych dyscyplin, jakość ksztatcenia, metodologia (Die Familienwissenschaften-ihr Platz unter den anderen Disziplinen, die Qualität der Ausbildung und die Methodologie), der von H. H. Prof. Dr. habil. Józef Stala geleitet wurde, konnten folgende Referenten gewonnen werden: 1. Prof. Dr. habil. Wojciech Krzysztof Świątkiewicz (UŚ), Kierunek nauki o rodzinie (Die Ausrichtung der Familienwissenschaften); 2. Prof. UKSW Dr. habil. Elżbieta Osewska (UKSW), Troska o jakość ksztatcenia na kierunku nauki o rodzinie (Die Sorge um die Qualität der Ausbildung auf die Familienwissenschaften hin); 3. H. H. Prof. Dr. Christian Gostečnik, Dozentin Dr. Barbara Simonič, Tanja Pate (Universität Ljubljana, Slowenien), Relational Family Therapy in theory and practice (Beziehungsorientierte Familientherapie in Theorie 
und Praxis); 4. Prof. Ateneum Dr. habil. Małgorzata Stopikowska (Ateneum - Hochschule Danzig), Nauki o rodzinie w Polsce i na świecie - koncepcje $i$ perspektywy rozwoju (Familienwissenschaften in Polen und der Welt - Konzeptionen und Entwicklungsperspektiven).

Die Teilnehmer des zweiten Konferenztags unterstrichen, dass die Familienwissenschaften eine neue Wissenschaftsdisziplin darstellen, weshalb sie vor der Herausforderung stehen, eine spezifische Methodologie auszuarbeiten. Die Familienwissenschaften greifen die integrale Problematik von Ehe und Familie auf, indem sie die humanistische Perspektive (die sich auf das Wesen von Ehe und Familie konzentriert) mit der gesellschaftlichen verbinden (welche die Erziehungsumfelder, die mit dem Ehepaar und der Familie zusammenarbeitenden Institutionen sowie diejenigen Systeme betrifft, die den Ehepartnern und der Familie bei der Realisierung ihrer Funktion Hilfe zukommen lassen).

Es ist zu betonen, dass sich in dieser Konferenz die Sorge der Organisatoren um die Familie, um alle ihre Mitglieder und um die Verwirklichung ihrer grundlegenden Funktionen ausdrückte. Dies verdeutlichte auch H. H. Prof. Dr. habil. Władysław Zuziak, Rektor der UPJPII in seinem Brief: „Im Namen des Senats und der gesamten akademischen Gemeinschaft der Päpstlichen Universität Johannes Paul II. in Krakau möchte ich den verehrten Professoren anlässlich der Organisation der zehnten internationalen Konferenz Wspótczesna rodzina: perspektywy lokalne i europejskie meine herzlichen Glückwünsche aussprechen, zu der sich Vertreter der Familienforschung aus Deutschland, Tschechien, Slowenien, Polen und aus der Slowakei versammelt haben. Mit großer Freude habe ich der Bitte entsprochen, die Schirmherrschaft zu übernehmen... Aus ganzem Herzen danke ich für das Aufnehmen eines so bedeutenden Problemkreises, für die Organisation der heutigen Konferenz und für das Engagement, die Thematik der Familie weiter $\mathrm{zu}$ verbreiten... Ebenso gratuliere ich $\mathrm{zu}$ der ausgezeichneten Zusammenarbeit mit den Hochschulen und Universitäten außerhalb Polens. Anlässlich des Europäischen Symposiums der Universitätsdozenten (2004) nannte der selige Johannes Paul II. die Familie den 'Spiegel der Gesellschaft', der jedes ihrer Probleme zurückwirft. Es ist offensichtlich, dass alle Auswirkungen der Umbrüche in Osteuropa auf die Familie Einfluss nehmen, wie z. B. die Migration von Erwerbstätigen oder länderübergreifende Eheschließungen. Darum ist es umso wichtiger, die in der Familie vor sich gehenden Veränderungen zu beobachten und auf wahrnehmbare Schwächen 
schnell zu reagieren... Ich bin sehr dankbar dafür, dass sich die Päpstliche Universität Johannes Paul II. in Krakau dank der Initiative ihrer Mitarbeiter so aktiv in die bedeutungsvolle gesellschaftliche Diskussion über die Lage der Familie einbringt. Noch einmal gilt mein Glückwunsch den Organisatoren für die Auswahl der Thematik. Ich wünsche allen fruchtbringende und bereichernde Besprechungen".

Die Konzeption für die Konferenz entstand während einer Vielzahl akademischer Begegnungen, die in Slowenien (Ljubljana, Maribor), in Tschechien (Prag) und in Polen (Warschau, Krakau und Tarnów) stattfanden, wie auch im Verlauf von Gesprächen mit Vertretern von Familienbewegungen und -verbänden, mit Studenten und Eltern. Die thematisch vielfältigen Vorträge und die lebhaften Diskussionen während der Konferenz brachten die immense Sorge um die moderne Familie und um die Disziplin der Familienwissenschaften deutlich zum Ausdruck. Alle Verantwortlichen für Familienwissenschaften waren sich einig, dass sowohl die Notwendigkeit als auch der Bedarf bestehen, gemeinsame Anstrengungen und Aktivitäten für die Sache von Ehe und Familie aufzunehmen, da diese sich gegenwärtig in einer Krisensituation befinden, in der ihre Fundamente gestärkt werden müssen. 